\title{
La Performance Teatral como Dispositivo Transferencial Crítico de la Violencia Micromachista en Adolescentes
}

\section{Theatrical Performance as a Transferential Device Critical of Micro- Chauvinisms Violence in Adolescents}

\author{
Rocío Anguita-Martínez *, Yasna Pradena-García, Eduardo Fernández-Rodríguez \\ Universidad de Valladolid, España
}

DESCRIPTORES:
Teatro aplicado
Educación secundaria
Promoción de la
igualdad de género
Modelo actancial
Competencia
espectatorial

\section{KEYWORDS:}

Applied theatre

Secondary education

Gender equality

promotion

Actantial model

Spectatorial

competence

\begin{abstract}
RESUMEN:
Este artículo analiza la implementación de una performance teatral para la prevención de la violencia micromachista y la promoción de la igualdad creada por estudiantes de Formación Profesional y dirigida al alumnado de Secundaria. Partiendo de una metodología cualitativa de investigación basada en el estudio de casos, este trabajo se centra en: a) analizar los roles actanciales de los personajes que representan situaciones de micromachismos en la adolescencia; b) comprender el proceso de mediación pedagógica que realiza el alumnado de FP tras la representación de la obra para facilitar el distanciamiento cognitivo y recuperar los conocimientos tácticos de género de la audiencia; y c) describir la segunda representación de la obra modificada en la mediación pedagógica para presentar alternativas de mejora en el contenido. Los resultados nos permiten concluir sobre las ventajas de los modelos actanciales para diagnosticar la actualidad del micromachismo juvenil; las particulares características de la performance teatral como experiencia en la que la juventud se ve representada e interpelada respecto de la violencia de género; y por último, las posibilidades que ofrece el propio espacio teatral en tanto laboratorio de creación ciudadana donde ampliar la competencia espectatorial adolescente con perspectiva de género.
\end{abstract}

\begin{abstract}
:
This article analyses the implementation of a theatrical performance for the prevention of micro-chauvinisms violence and the promotion of equality created by vocational training students and aimed at secondary school students. Using a qualitative research methodology based on case studies, this work focuses on: a) analysing the acting roles of the characters that represent situations of micro-chauvinisms violence in adolescence; b) understanding the process of pedagogical mediation carried out by the VET students after the performance of the play to facilitate cognitive distancing and recover the audience's tactical knowledge of gender; and c) describing the second performance of the play modified in the pedagogical mediation to present alternatives for improving the content. The results allow us to conclude on the advantages of actantial models to diagnose the actuality of youth micro-chauvinisms violence; the particular characteristics of the theatrical performance as an experience in which young people are represented and challenged with respect to gender violence; and finally, the possibilities offered by the theatrical space itself as a laboratory of citizen creation where to expand the competence of adolescent spectatorship with a gender perspective.
\end{abstract}

CÓMO CITAR:

Anguita-Martínez, R., Pradena-García, Y. y Fernández-Rodríguez, E. (2021). La performance teatral como dispositivo transferencial crítico de la violencia micromachista en adolescentes. Revista Internacional de Educación para la Justicia Social, 10(2), 27-42. https://doi.org/10.15366/riejs2021.10.2.002

${ }^{*}$ Contacto: rocio.anguita@uva.es

ISSN: 2254-3139

revistas.uam.es/riejs 


\section{Introducción}

Un ámbito especialmente preocupante en el que se manifiestan la pervivencia de los roles tradicionales y sexistas en las relaciones entre hombres y mujeres es en el de la población adolescente y joven. Son diversos los estudios que señalan la creciente normalización de la violencia de género trasladando e imponiendo a sus interacciones toda una serie de valores hegemónicos relacionados con el patriarcado (Ballesteros et al., 2018; Díaz-Aguado et al., 2020) a través de una serie de prácticas sutiles y cotidianas de dominación masculina, control parental y violencia simbólica o invisible denominadas como micromachismos (Bonino, 1995, 1996; Donoso et al., 2018; García et al., 2018; Román y Faepb, 2017) que atentan contra la autonomía personal de las mujeres y que suelen ser invisibles o, incluso, estar perfectamente legitimadas por el entorno social.

El concepto de Teatro Aplicado (Nicholson, 2005; Sedano-Solís, 2019) emerge dentro del ámbito más global de los estudios teatrales como un término para describir diversas actividades que utilizan el teatro como una propuesta orientada a facilitar la expresión creativa de sus participantes en contextos educativos, institucionales y comunitarios (García-Huidobro et al., 2021; Motos y Ferrandis, 2015), con el objetivo de analizar y comprender situaciones de su vida diaria, capacitándoles para el desarrollo de una ciudadanía más igualitaria e inclusiva y permitiéndoles plantear nuevas formas expresivas y reflexivas vinculadas con la interacción entre performers y los diversos públicos (Balme, 2013), sobre todo en lo que concierne al rol de los primeros en tanto facilitadores o mediadores del aprendizaje (Motos et al., 2013; Santos, 2017).

Si hay un ámbito especialmente relevante para valorar el papel que puede jugar la dramatización teatral como herramienta de concientización, empoderamiento y transformación social es el vinculado a la mujer y el género (Gallagher y Rodricks, 2017). La dramatización teatral se transforma en un recurso creativo y estético que facilita la visibilización y comprensión de problemáticas como la discriminación, las opresiones, la desigualdad y/o la violencia en contra de las mujeres (Sales Oliveira, et al., 2019). La utilización del teatro como herramienta didáctica para la prevención de la violencia de género y la promoción de igualdad, no sólo ayuda a simbolizar situaciones injustas y opresoras de la vida cotidiana para poder comprenderlas, cambiarlas y mejorarlas $(S t a h l, 2018)$, sino que también hace referencia a la potencialidad que tiene como recurso de transformación individual, colectiva y social (Taylor, 2019).

En este marco, es especialmente relevante analizar el rol social que cumplen las y los diferentes personajes en las escenificaciones teatrales relacionadas con la violencia de género. La literatura científica cuenta ya con suficiente bibliografía respecto de la aplicación al teatro del modelo de semiótica narrativa basado en roles actanciales (De Toro, 1995; Ubersfeld, 1999), así como de los trabajos en semiótica de la recepción teatral que rescatan el hacer empírico de las y los espectadores y el papel central que juegan las dimensiones emocionales y cognitivas en la acogida del texto representado a nivel escénico (Helbo, 2013; De Marinis, 2005; Mirza, 2011; Pavis, 2017).

Especialmente de interés para este trabajo son las relaciones y oportunidades de la dramatización teatral para el ejercicio de prácticas dialógicas y de concientización crítica (Villanueva Vargas, 2019). Partiendo de planteamientos influidos por la pedagogía freireana, las y los estudiantes a través de prácticas escénicas reconocen y cuestionan sus propias situaciones de opresión y exploran nuevos espacios para la justicia social (Giambrone, 2016). En este sentido, la aplicación de experiencias de teatro performativo como metodologías innovadoras para la prevención de la violencia de género y la promoción de la igualdad en el ámbito educativo (Gallagher y Sahni, 2019) se convierten en laboratorios de investigación creativa (Besselink, 2013) al trabajar sobre un conocimiento corporalizado, localmente situado y distribuido culturalmente (Davids y Willemse, 2014) en los que adolescentes jóvenes ven escenificado y re-creado en relación con situaciones de micromachismo, y que les ayuda a la hora de generar procesos de concienciación, empoderamiento, comunicación e interacción social (Motos et al., 2018). 


\section{Método}

\subsection{Contexto del estudio y selección de participantes}

Esta investigación se desarrolla con un enfoque cualitativo de estudio de casos (Stake, 1999) que se realiza a través de la observación no participante, la descripción y el análisis de las presentaciones de una performance teatral creada para la prevención de la violencia de género y la promoción de la igualdad. Asimismo, esta experiencia educativa-artística se complementa con una mediación o dinamización que se realiza con la audiencia de estudiantes de educación secundaria tras la presentación de la obra.

Esta es una experiencia particular de prácticas que se pone en marcha durante el primer trimestre del curso académico 2018/19 en el contexto de un Instituto público de Educación Secundaria de una ciudad de Castilla y León, concretamente con el alumnado de dos Ciclos Formativos de Grado Superior de Formación Profesional, de Técnico Superior de Animación Sociocultural y Turística (AST) y Promoción de la Igualdad de Género (PIG) y en el marco de tres asignaturas diferentes de las dos titulaciones: Habilidades Sociales ( $2^{\circ}$ curso de PIG), Prevención de la Violencia de Género (10 curso PIG) e Información Juvenil (20 curso de AST) con el trabajo conjunto de las tres docentes responsables de las asignaturas. Por tanto, la implementación de esta experiencia educativa artística teatral se lleva a cabo con los tres grupos conjuntamente, con un total de 45 estudiantes que participan en distintos roles de la performance teatral y la dinamización.

El resultado de dicho proceso educativo-artístico es la obra de Teatro Aplicado "Da la vuelta al espejo" cocreada, representada (actores/actrices) e implementada por el alumnado de Formación Profesional guiado por las profesoras encargadas. Esta performance teatral, ha sido presentada en seis ocasiones a una audiencia de estudiantes de educación secundaria de diversos institutos públicos de Educación Secundaria, en cinco centros cívicos de la ciudad. La propuesta de trabajo en torno a la performance teatral incluye los siguientes momentos: a) el alumnado de FP, en sus roles de actores y actrices, presentan escenas dramatizadas vinculadas con situaciones cotidianas de micromachismos vividas por mujeres adolescentes (Figura 1); b) una dinamización que se realiza tras la presentación de la obra, guiada por el alumnado de FP, en su rol de mediadoras/es, en la que forman grupos con la audiencia de estudiantes de secundaria y, a través del diálogo y perspectiva crítica de género, reflexionan acerca del contenido que se presenta en la obra; c) la creación de nuevas escenas, que surgen en el diálogo de la dinamización, en las que se modifican algunos contenidos de la obra teatral orientados por la búsqueda de relaciones igualitarias entre jóvenes adolescentes, la autonomía femenina o una nueva condición masculina que rompa con los valores patriarcales.

Figura 1

Esquema de la performance teatral

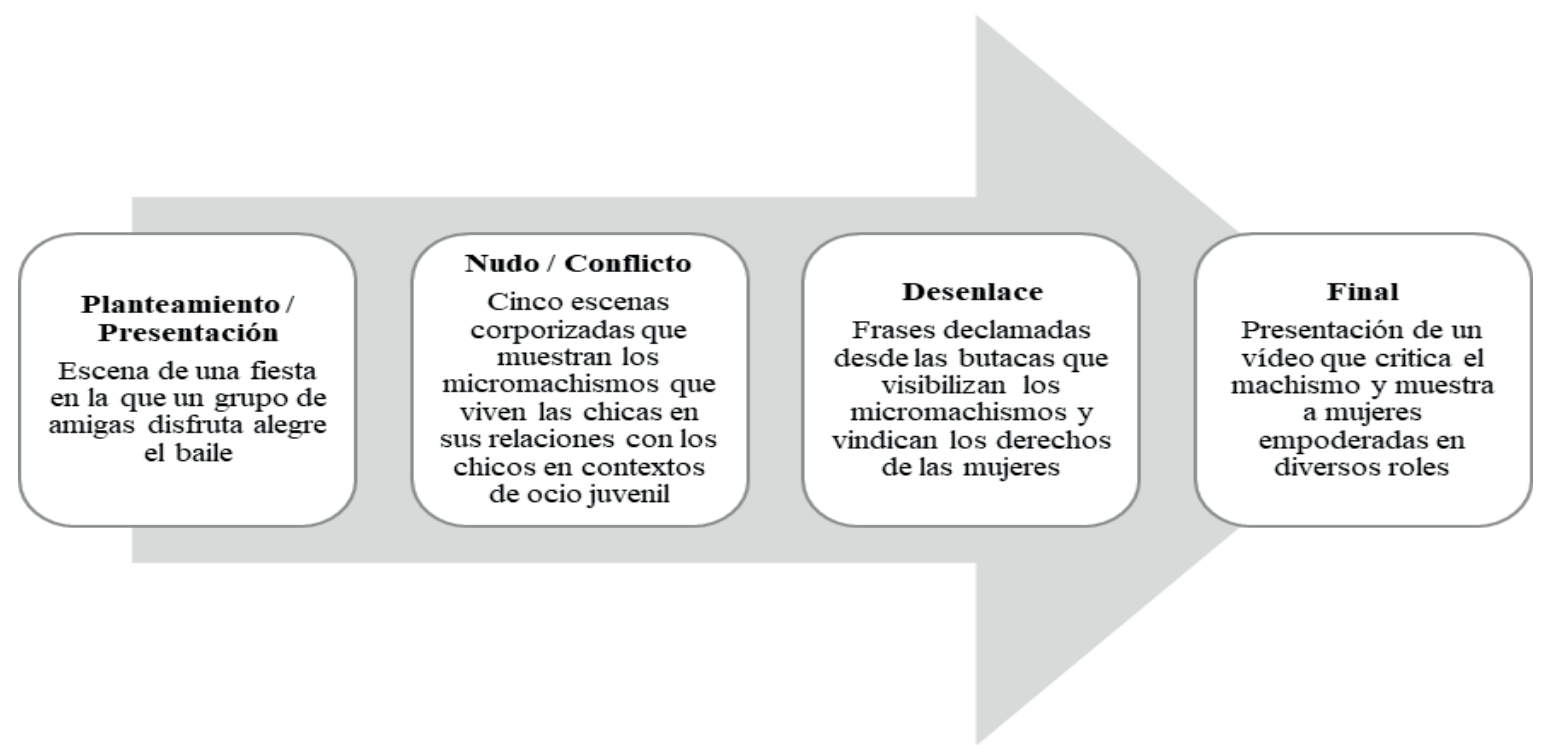




\subsection{Objetivos de investigación, foco de estudio y dimensiones de análisis}

El objetivo de esta investigación ha sido conocer la potencialidad del Teatro Aplicado para la prevención de la violencia de género y la promoción de la igualdad a través de las presentaciones de la performance teatral "Da la vuelta al espejo" creada por estudiantes de Formación Profesional (FP), y dirigida a una audiencia de estudiantes de Educación Secundaria (ESO) (Cuadro 1). Más en concreto, se pretendía:

- Analizar los roles actanciales que juegan tanto hombres como mujeres en la reproducción de determinadas conductas y estrategias relacionales asociadas al micromachismo, valorando el efecto de la dramatización escenificada a la hora de generar una experiencia colectiva y compartida capaz de activar procesos de interpelación entre la audiencia de estudiantes de secundaria.

- Comprender el papel que juegan los procesos de mediación pedagógicas contemplados en la propia performance teatral a la hora de favorecer no sólo la activación de procesos perceptivos, afectivos y cognitivos entre el alumnado de secundaria a través del diálogo participativo respecto de las diversas escenas de micromachismos, sino también de las estrategias implementadas para facilitar una reflexión compartida respecto de los conocimientos tácitos de género que traen consigo las y los estudiantes de secundaria.

- Describir y analizar los procesos de enunciación colectiva y coautoría que se establecen a través de la performance teatral, y las formas en las que se desarrolla en la audiencia de secundaria una competencia espectatorial con enfoque de género a través de su implicación en la generación de nuevas producciones narrativas a partir de la representación inicial escenificada por las y los actores/estudiantes de Formación Profesional.

Cuadro 1

Preguntas y categorías para el análisis de la dinamización y la recreación

\section{Preguntas de investigación \\ Categoría de análisis}

Eje del deseo: motivaciones y propósitos que animan las acciones de mujeres y hombres adolescentes en contextos de violencia micromachista

¿Cuáles son los roles actanciales impli- Eje de la comunicación: valores e ideologías antagónicas implicadas en cados en la performance teatral " $\mathrm{Da}$ la la producción narrativa (empoderamiento femenino versus reproducvuelta al espejo"? ción cultura patriarcal)

Eje del poder: acciones, personajes y mecanismos que permiten o inhiben la emancipación y autonomía de las mujeres adolescentes en contextos de socialización y ocio juvenil

¿Qué estrategias de mediación pedagógica son activadas para facilitar en la audiencia procesos de involucramiento respecto de la violencia micromachista?

Pedagogías dialógico-reflexivas para la implicación afectiva de las escenas representadas

Mecanismos de facilitación para la comprensión de la obra

Problematización de los conocimientos tácitos de género

¿Qué procesos de enunciación colectiva se establecen a partir de la performance teatral con la audiencia?

Creaciones narrativas generadas por la audiencia

Desarrollo de la competencia espectatorial con enfoque de género

\subsection{Instrumentos y procedimiento de recogida de datos}

En la realización del estudio las investigadoras utilizaron instrumentos, procedimientos y técnicas etnográficas cuyo objetivo era la búsqueda de una descripción densa del escenario seleccionado, garantizando además la profundidad en el análisis de los datos cualitativos y lograr la validez y credibilidad en nuestras proposiciones teóricas generadas. Para una mayor comprensión de la utilización de los instrumentos y procedimientos hemos realizado una síntesis en el Cuadro 2, que relaciona cada instrumento con los procedimientos que hemos llevado a cabo durante el proceso. 
Cuadro 2

Procedimientos de recogida de datos de las Observaciones audiovisuales de representaciones

\begin{tabular}{llll}
\hline Sesiones & \multicolumn{1}{c}{ Procedimientos } & \multicolumn{1}{c}{ Datos } & Codificación \\
\hline Función 1 & & $\begin{array}{l}\text { Reflexión grupal en equi- } \\
\text { pos de trabajo }\end{array}$ & F/ RG \\
Función 2 & Observación (no) participante realizada por dos & Producción narrativa de & F / PM \\
Función 3 & investigadoras del montaje teatral & las estudiantes & \\
Función 4 & Grabaciones en video & $\begin{array}{l}\text { Propuesta narrativa de los } \\
\text { estudiantes }\end{array}$ & F/PH \\
Función 5 & & Función 6 &
\end{tabular}

El procedimiento para el análisis de datos lo hemos centrado en la revisión de las grabaciones de las seis presentaciones que se realizaron de la performance teatral en cinco centros cívicos. Este conjunto de registros audiovisuales ha sido organizado por medio del CAQDAS (Computer Assisted Qualitative Data Analysis Software) (Sánchez, 2008) NVivo 12 Plus, con el objetivo de facilitar la visualización del contenido de los registros, su transcripción, codificación y análisis. Las transcripciones de datos verbales corresponden a los comentarios que han realizado mediadores/mediadoras conjuntamente con estudiantes de la audiencia tras la presentación de la performance teatral en la etapa de dinamización grupal. Igualmente, hemos transcrito las aportaciones que hacen los grupos a la performance teatral para una segunda representación en la que recrean una realidad transformada.

Para realizar este estudio, se contó con el permiso, la confidencialidad y el anonimato de todas las personas e instituciones participantes en esta investigación. Para el acceso al centro educativo de educación secundaria se pidió autorización por escrito a la Dirección General de Innovación Educativa de la Consejería de Educación de la Junta de Castilla y León y, posteriormente, a la dirección del centro, así como se le comunicó a la inspección educativa. Una vez cumplidos estos trámites, se negoció el acceso y las condiciones de la investigación con los tres grupos de estudiantes y las tres profesoras en una sesión presencial y luego se firmó de forma individual el consentimiento informado de participación para todo el proceso de investigación. Igualmente se pidió permiso a todos los grupos de educación secundaria que participaron como espectadores de las representaciones, tanto para la grabación como para el uso posterior de datos.

\section{Resultados}

\subsection{La performance teatral como dispositivo transferencial crítico de la violencia micromachista: Análisis de roles actanciales}

La performance teatral "Da la vuelta al espejo" está compuesta por pasajes narrativo-visuales relacionados con la violencia de género específicamente los micromachismos. Estos pasajes, han sido analizados siguiendo el modelo actancial (Greimas, 1971) como se puede ver en una adaptación en la Figura 2. 
Figura 2

Esquema adaptado de roles actanciales de Greimas

\section{ESQUEMA ROLES ACT ANCIALES}

Destinador
¿Qué o quiénes impulsan
la acción?
Visibilización y
concientización sobre los
micromachismos

\section{Ayudante}

¿Qué o quiénes colaboran

para lograr el objeto?

1. Frases declamadas que

visibilizan los micromachismos

$\mathrm{y}$ vindican los derechos de las jóvenes

2. Canción y vídeo relacionados con la temática de la obra

3. Elemento simbólico de

espejo que refleja la realidad e interpela a la audiencia
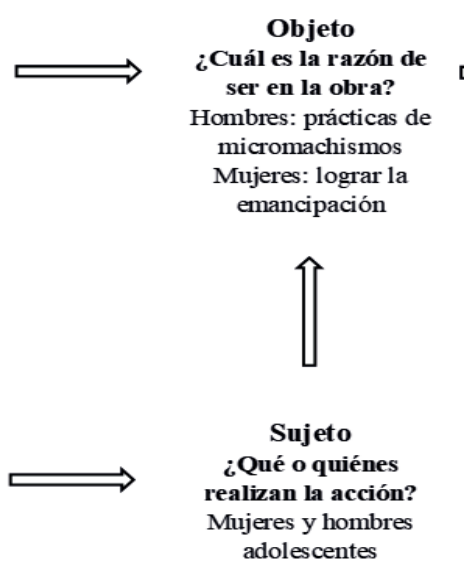

\author{
Destinatario \\ ¿A quiénes se quiere \\ Capacitar a la juventud \\ en la detección y \\ erradicación de \\ situaciones de \\ micromachismos
}

\section{Oponente} ¿Qué o quiénes se oponen al objeto? Hombres: mecanismos coercitivos Mujeres: estrategias de empoderamiento de las

A partir de este esquema, se identifican tres categorías actanciales (eje del deseo, eje de la comunicación y eje del poder) y seis roles o funciones: sujeto-objeto-destinador-destinatario-oponente-ayudante. En la estructura actancial: "un sujeto (S) desea un objeto (O)"; "es ayudado por un ayudante (Ay) y orientado por un oponente) (Op)"; "el conjunto de los hechos es deseado, orientado, arbitrado por un destinador (D1) en beneficio de un destinatario (D2)", aplicando a la performance teatral diríamos entonces que:

- El eje del deseo (sujeto-objeto): traza la trayectoria de la acción y la búsqueda del protagonista, ya sea de las mujeres jóvenes que sufren situaciones de micromachismo o de los varones jóvenes que ejercen dichas prácticas de violencia patriarcal. En el primer caso, buscando una apropiación femenina del espacio público y de su independencia (Figura 3).

Figura 3

Comienzo de la obra: Baile de chicas

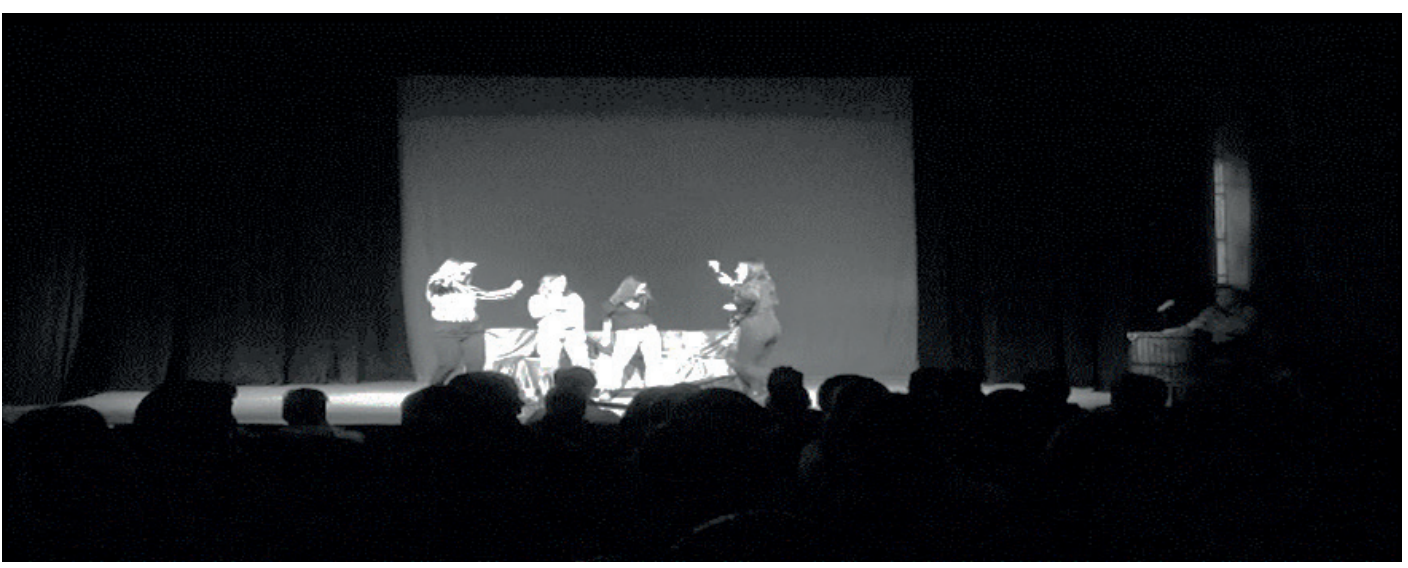

Mientras que, en el segundo caso, los jóvenes buscarán mantener el dominio y control evitando todo posibilidad de rebelión y resistencia de la mujer a quien considera desde una lógica de inferioridad (Figura 4). 


\section{Figura 4}

Representación de la masculinidad hegemónica por sobre la mujer

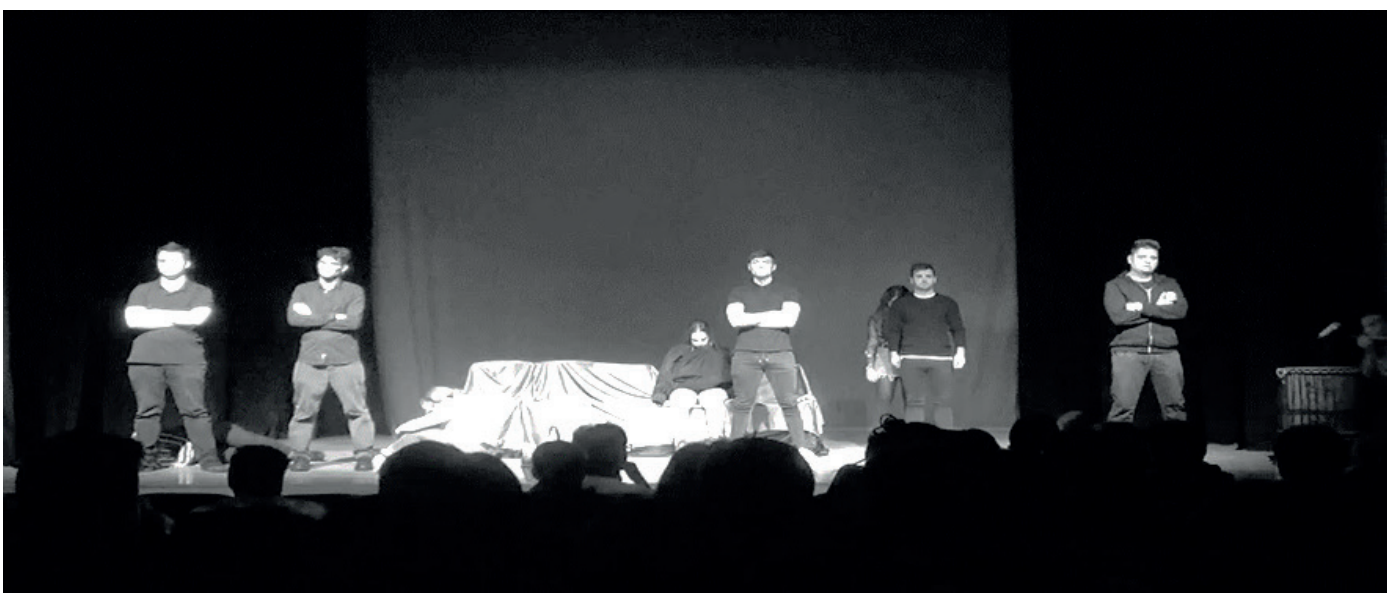

- El eje de la comunicación (destinador-destinatario): es el del control de los valores y por ende de la ideología que anima la producción narrativa, ya sea en la línea de dar visibilidad, detectar y erradicar toda esa serie de microviolencias ejercidas en contextos de ocio y socialización por varones adolescentes, o en la búsqueda de mecanismos de concientización que faciliten el empoderamiento femenino y la asunción de nuevos roles masculinos para una sociedad más igualitaria.

- El eje del poder (ayudante-opositor): facilita o impide la comunicación y el cambio social. Produce las circunstancias y las modalidades de la acción, que, en este caso, es representado por elementos simbólicos como frases declamadas que emergen desde las butacas y espejos que reflejan la realidad.

¿Y si le diéramos la vuelta al reflejo de esta sociedad y por ejemplo mañana ninguna de nosotras tuviéramos miedo de ir a cualquier hora a casa solas?

Paralelamente a la declamación de frases, "los espejos" se van ubicando bajo el escenario frente al público, “dando vuelta el espejo” y enseñando letras que más tarde construirán una frase. Las siguientes frases dicen “¿Y si mañana pago yo?”

¿Y si sabes que para él solo eres un juego, porque no le dices game over?

¿Y qué pasa si yo ya no quiero comportarme como una señorita?

Decid lo que queráis, ellas harán lo que les dé la gana

¿Si una mujer te dice que no, por qué no lo aceptas?

¿Y si le digo a mi pareja que nunca me ha gustado que me llame churri?

¿Y si mañana yo fuera la jefa de todos vosotros?

El problema no es su falda, sino de quien quiere levantarla

¿Qué pasa si no quiere darte la contraseña de su teléfono?

¿Y si yo decido cómo me visto y con quién me desvisto?

Bailo así porque me gusta no para provocar

¿Y si yo decido cómo y con quién vivir?

Me esforzaba en arreglarme hasta que me di cuenta que no estaba rota

Decían que necesitaba un héroe, y ella en uno se convirtió

¿Y si la del lado fuera mi compañera y no mi competencia? 
Estas frases permiten identificar toda una serie de mecanismos coercitivos o encubiertos de violencia micromachista en la socialización adolescente y también examinar una serie de mensajes sociales lanzados en redes de comunicación que reproducen modelos hegemónicos de masculinidad. Pero al mismo tiempo, también este eje da cuenta de todas aquellas expresiones de sororidad, acciones de resistencia activa y estrategia de empoderamiento que son utilizadas por las mujeres para recuperar el control de sus vidas y su autonomía.

\subsection{Mediaciones pedagógicas que facilitan el involucramiento de la audiencia adoles- cente para problematizar la violencia de género micromachista}

La dinamización es la etapa que se desarrolla tras la presentación de la performance teatral. En este momento, el alumnado de FP que ha puesto en marcha la obra cumple el rol de mediador o mediadora reuniéndose y formando grupos con la audiencia (estudiantes de secundaria), ya sea para activar la reflexión en torno a la obra, facilitar la comprensión respecto de algunos elementos de la obra que no han sido comprendidos por la audiencia, o sencillamente propiciando el debate entre los y las adolescentes en los que rescatar y confrontar los conocimientos tácitos de género que traen consigo fruto de sus propias experiencias personales y biográficas.

\section{a) Reflexionando y dialogando sobre las escenas representadas...}

La activación del diálogo y la reflexión son estrategias que utilizan mediadoras/es para activar en la audiencia las primeras percepciones y opiniones sobre la obra. Estas estrategias consisten en dar una relectura a la performance teatral desde distintas perspectivas y análisis crítico: comparando y ejemplificando la temática de la obra con otros hechos de la realidad; cuestionando ¿qué se sabe?, ¿qué se entiende?, ¿qué se siente?; valorando las experiencias e intereses vividos por la audiencia. La estrategia de comparación y ejemplificación con la realidad queda reflejada en la siguiente viñeta. En este caso, la mediadora además de ejemplificar hace un análisis crítico e interpela al estudiante sobre esta acción cotidiana que demuestra los estereotipos de género que se viven a diario.

Mediadora: Por ejemplo, en educación física, imaginaos que os mandan dos grupos para hacer equipo ¿tú a quién elijes primero a una chica o un chico?

Estudiante ESO (chico): A un chico porque pienso que va a ser mejor para el equipo porque tiene más condición física y el deporte es así. (F5RG1)

\section{b) Facilitando la comprensión de la obra...}

Los y las mediadoras facilitan la comprensión de aquellos elementos que la audiencia no ha logrado visualizar, que no han aparecido en el diálogo y/o que no entienden. En este caso, vemos que el diálogo se vuelve menos espontáneo y más "guiado". En otras palabras, cumplen la función de informar algo o enseñar los aspectos que no se conocen de la temática de la performance teatral.

\section{Mediadora: Os voy a hacer una pregunta: ¿Qué es el feminismo? \\ Estudiantes ESO (chica): Igualdad entre los dos géneros, entre hombre y mujer. (F2RG2)}

También surgen dudas sobre los aspectos estéticos metafóricos de la obra tales como los elementos de la "manzana" o los "espejos". En estos casos, el rol de la mediadora/or es explicativo y ejemplifica con otras situaciones cotidianas el significado de cada signo o concepto.

Mediadora: ¿Habéis entendido lo que representaban los espejos?

Estudiante ESO (chica): Es como el reflejo de lo que no se ve.

Mediadora: Es como el reflejo de la sociedad.

Mediador: Es como una manera de señalar, nosotros todos tenemos el papel para poder cambiarlo, porque al fin y al cabo todos somos cómplices de alguna manera, lo queramos o no. (F2RG4)

La facilitación es un espacio de transferencia de contenidos vinculados con la temática de género de la performance teatral, de diálogo y reflexión sobre esta problemática y de empatía que surge a través de la 
visualización de las historias que se narran en la obra, y la identificación de estas historias u otras similares en las experiencias personales que comparte la audiencia en el grupo.

\section{c) Problematizando los conocimientos tácitos de géneros de la audiencia...}

Un momento importante durante la dinámica de trabajo es cuando se problematizan algunas cuestiones relacionadas con los contenidos que traslada la performance en torno a la violencia micromachista. Para ello, se opta por estrategias de análisis grupal que favorecen la búsqueda en los conocimientos previos de la audiencia, lo que han entendido del mensaje de la obra y, sobre todo, lo que han sentido a nivel interno:

Mediadora: Las cinco chicas que estamos arriba sufrimos cada una, una forma de violencia de maltrato, de desprecio, de discriminación, como lo queráis llamar, y luego cogemos una manzana y la mordemos. ¿Con esto que os hemos dicho, qué creéis que puede representar? ¿Puede ser que al coger la manzana nos estemos empoderando? ¿Quécreéis?

Estudiante ESO (chicas): Se quedan pensado e intentan dar una respuesta.

Mediadora: ¿Qué significa empoderarse?

Estudiante ESO (chicas): Es como darle la vuelta a la tortilla.

Mediadora: ¿Habéis visto alguna escena o algunas de las frases en la que os hayáis visto reflejadas?

Estudiante ESO (chicas): Lo que han dicho del miedo cuando vuelves a casa, eso continuamente. (F3RG1)

Estas cuestiones llevan a una reconstrucción crítica de las experiencias e intereses vividos por la audiencia frente a la temática que se muestra. En este caso, la activación de la "memoria vivencial" en la audiencia se relaciona con un proceso interno de evocación y reconocimiento de la temática de la obra en sus propias vidas. La búsqueda en la memoria para dar respuesta a esas cuestiones que han surgido a través de la obra, su reflexión y verbalización reflejan que se siguen repitiendo prácticas de desigualdad y estereotipos de género presentes en la juventud, como queda patente en los comentarios que rescatamos a continuación:

Estudiante ESO (chica): Una cosa que yo puedo notar es tan sencilla con mirar un catálogo de juguetes, siempre nos dividen en una parte, rosa para las chicas con juguetes de cocina, princesas, $y$ lo de los chicos azul, y son súper héroes, bombero, policía. A lo mejor una chica quiere ser policía o un chico quiere ser cocinero. No hay que diferenciarlo por colores o por cosas que son de chicos o de chicas. (F2RG2)

Estudiante ESO (chica): Sí, por ejemplo, voy a contar un caso. Resulta que un amigo mío, se compró una funda que era rosa, del teléfono, le gustaba el rosa y se ha comprado una funda rosa. Y me comentó que la gente le hacía bromas, "iieres una tía!!” porque llevas una funda de color rosa. Y yo le dije, no hagas caso porque, si a ti te gusta y le queda bien al móvil, mira negro con rosa queda bien, ya está. (F2RG2)

\subsection{Procesos de enunciación colectiva y co-autoría con enfoque de género}

\section{a) Buscando creaciones narrativas por parte de la audiencia...}

Las propuestas de nuevas ideas desde una perspectiva crítica a la performance teatral surgen por la necesidad de responder de forma creativa a la problemática de la violencia de género imaginando otra realidad posible más igualitaria. Esta es una acción de reconocimiento y confianza en el conocimiento del espectador y espectadora como sujetos creativos y transformadores invitándoles a participar activamente en la modificación a nivel narrativo de la obra.

Mediadora 1: Estaría muy bien que los chicos os posicionarais con estas cosas.

Mediadora 2: Que deis un paso adelante, esta es una lucha de todos.

Estudiante ESO (chico): En general salen muchos artículos, por ejemplo, "a este hombre le ha agredido su mujery todos se han reído de él" (F2RG5) 
Por otra parte, el rol de mediadora/or se encuentra con diversos conflictos relacionados con las interacciones que suceden con la audiencia. Para ello, tienen que buscar en sus conocimientos previos de formación profesional técnicas de manejo grupal para mediar las diversas opiniones que surgen en el grupo frente a la obra:

Estudiante ESO (chica): No, yo no estoy de acuerdo con él, lo que menos pasa es que te miren el móvil.

Mediadora: ¿Y tú que crees que es lo que más pasa?

Estudiante ESO (chica): Yo pienso que a nuestra edad lo que más pasa es la del alcohol, las demás son para más mayores (Refiriéndose a las escenas) (F2RG1)

Al mismo tiempo, deben manejar conceptos relacionados con la temática de género para "enseñar" contenidos específicos. Con esto queremos decir que la mediadora/or tiene que estar preparada para enfrentar el contacto grupal y las opiniones que se encuentran en la diversidad de espectadores y espectadoras, así como también con la información que entrega:

Mediadora 2: ¿Sabes lo que es violencia de género?

Estudiante ESO (chico): Sí, de un hombre a una mujer.

Mediadora 2: Por el mero hecho de ser mujer. El otro tipo de violencia puede ser violencia familiar. (F2RG5)

Este momento de mediación dialógica, nos deja ver la comprensión del tema que se aborda en la obra por parte de la audiencia, el nivel de atención que se ha logrado en la recepción de la performance teatral, así como la atención a la diversidad de opiniones que han surgido a partir de la obra. Asimismo, deja vislumbrar el "deseo de entendimiento" y la disposición frente a quien se escucha. Todo esto como eje transversal para poner en común una problemática que vivimos día a día, pero que es invisibilizada o simplemente no estamos "sensibilizadas" para detectarla.

\section{b) Materializaciones narrativas corporizadas que favorecen la competencia espectatorial con en- foque de género}

En esta etapa, la audiencia corporiza las propuestas que han hecho previamente en la fase de búsqueda de nuevas narrativas. Estas, "recreaciones" corporizadas tienen como objetivo transformar la realidad de violencia de género que se presenta en la obra, agrupándolas en tres ejes temáticos: derecho a la emancipación de las mujeres (cuerpo, estética y acciones), prácticas igualitarias y nueva condición masculina, imaginando una realidad social emancipadora y horizontal en las relaciones de género

Derecho a la emancipación de las mujeres (su cuerpo, acciones y decisiones estéticas): En este eje, emergen diversas vindicaciones vinculadas a la emancipación de los cuerpos de las mujeres. "Hacer lo que se quiera con el cuerpo" se convierte en un axioma o imaginario que está condicionado por diversos factores sociales y éticos, tanto para hombres como para mujeres. Sin embargo, en el comentario que incorporamos abajo, la estudiante se refiere a un hecho que es reiterativo en la "opinión pública patriarcal" frente a las acciones de una mujer cuando hace "lo que quiere" con su cuerpo. Si una mujer es libre con su cuerpo es: guarra, facilona, sucia, perra, prostituta, entre otras denominaciones que insultan y coartan la autonomía de la mujer:

\section{¿Porqué por hacer lo que quiera con mi cuerpo tengo que ser una guarra? (F2PM11)}

Por otra parte, vemos una serie de producciones narrativas en línea de un cuestionamiento crítico sobre el reconocimiento y respeto al cuerpo femenino "tal y como es", rompiendo los estereotipos estéticos heredados socialmente como la depilación. Así como de la libertad de las mujeres a decidir cómo verse. También emergen creaciones narrativas que se centran en la identificación e interpelación hacia las opiniones éticas que se generan sobre algunas acciones que las mujeres realizan y que son "mal vistas":

¿Y si nacemos con pelos porqué tenemos que quitárnoslo? (F6PM16)

¿Por qué me tengo que vestir como tú quieras y no como yo decida? (F1PM2)

¿Y si no quiero llevar sujetador? (F5PM7) 
En todos estos casos, las estudiantes interpelan a una sociedad machista a la que se le ha permitido opinar sobre qué tipo de acciones son o no pertinentes para las mujeres en cuanto a sus cuerpos, formas de auto-representación o formas de comportarse.

Prácticas igualitarias y nueva condición masculina: En este eje, la audiencia aporta narrativas sobre las prácticas sexistas y desiguales que perciben en sus propias vidas o en sus entornos más cercanos. En estas creaciones podemos deducir que los y las adolescentes se sienten excluidas y excluidos de algunas prácticas de tipo deportivas o artísticas porque siguen siendo mayoritariamente "de hombres" o "de mujeres". Y que los contextos socioculturales siguen siendo sexistas segregando a las mujeres de prácticas vinculadas a "lo masculino" y a los hombres de "lo femenino".

$$
\begin{aligned}
& \text { ¿Y si jugamos al futbol, ¿qué pasa? (F2PM5) } \\
& \text { ¿Por qué no puedo bailar ballet? (F1PH3) }
\end{aligned}
$$

En otro sentido, y respecto de los comportamientos igualitarios, podemos examinar la siguiente producción narrativa:

Queremos resaltar que en el vídeo nos ha parecido muy chulo eso de que sean las mujeres las que representan el sexo superior. Y nuestra frase es: "No son lo que está entre sus piernas, sino lo que está entre sus orejas". Y hemos hablado sobre algunas situaciones de machismo cercano de alguna amiga y un adulto borracho que se la han ido las manos, o alguna amiga que su novio la controlaba. (F2PH9)

En este caso, el estudiante valora el recurso mediático (vídeo musical) que presenta a mujeres empoderadas. También, vindica la intelectualidad y critica la visión estereotipada de la "mujer-contenedor-vagina". Asimismo, evoca situaciones vividas por sus amigas para visibilizarlas en el contexto corporizado como "denunciante" y hombre "no cómplice" de estos hechos.

Crear conciencia sobre el anquilosado rol masculino patriarcal, entre el estudiantado fomenta la capacidad de repensar otras formas de comportamientos masculinos, para "imaginar", desde la metáfora teatral, otras masculinidades más igualitarias y respetuosas. En el comentario que señalamos abajo, el estudiante hace un ejercicio de autoconciencia frente a su comportamiento con una mujer en el caso que ella decida "no hacer algo".

Yo como hombre no puedo decir lo que tiene que hacer una mujer si ella no quiere. (F4PH3)

En ese mismo sentido, vemos cómo algunas estudiantes son capaces de objetivar alguno de los obstáculos con los que los varones se encuentran al momento de querer romper los estereotipos de género que les han sido asignados por el patriarcado:

Las mujeres tienen un papel importante, pero el machismo no solo afecta a la mujer, afecta al hombre, pero de una manera distinta, la visualización del hombre como poderoso, eso hace que un chico... quiero ayudar a planchar, a mi me gusta diseñar, me gusta la ropa, me gusta el rosa... (maricón, débil) todas estas cosas ¿Y eso no machismo? Eso también es machismo. La discriminación va para ambos bandos, lo único que la mujer lo sufre de una manera bastante más brutal. (F5PM11)

Imaginando una realidad social emancipadora y horizontal en las relaciones de género: La visualización de una nueva realidad se manifiesta desde distintas perspectivas entre la audiencia que participa en la obra. Por una parte, emerge la sororidad, como acción de mutualidad crítica entre mujeres en respuesta a los abusos que viven, siendo conscientes además de la necesidad de apoyo. En estas producciones narrativas subyace la necesidad de ayuda entre mujeres, en oposición a lo que se propone desde una visión patriarcal donde priman acciones y relaciones basadas en la rivalidad y la competitividad. Se reivindica, por tanto, la necesidad de trabajar la sororidad para fomentar el agrupamiento femenino sano, fortaleciendo las redes de apoyo y los cuidados.

\section{¿Por qué tengo que llamar a una amiga para sentirme segura? (F6PM4)}

Queremos recalcar el mensaje de que no tenemos que competir entre nosotras porque algo muy importante es que tenemos que apoyarnos porque ¿si no lo hacemos nosotras...? (F2PM8) 
Al mismo tiempo, surgen los reclamos hacia una sociedad que perpetúa la cosificación de la mujer y por medio de estos, la conciencia de querer cambiar esa realidad. Las creaciones narrativas que exponemos a continuación responden a la conciencia de las jóvenes sobre el cuerpo de la mujer como objeto que se puede utilizar de distintos modos. Ellas se posicionan frente a esta cosificación y se niegan a continuar con ese rol que se les ha atribuido históricamente a las mujeres (mujer-cosa / mujer-mascota / mujer-procreadora):

\author{
Nosotras somos más que sexo. (F2PM10) \\ ¿Y si no fuera tuya ni de nadie? (F4PM2) \\ No soy una perra, no necesito collar. (F5PM6) \\ Soy una persona, no soy un muñeco. (F5PM7)
}

Paralelamente, van apareciendo posicionamientos relacionados con asuntos como el de la racialidad, inspirados en este caso en la presentación del vídeo musical que aparece al finalizar la obra teatral en la que muestran a "mujeres racializadas". De un lado, hay un interés por mirar la violencia de género más allá de posiciones etnocéntricas y hegemónicas vinculadas con el feminismo:

Todavía hay muchas ideas sobre que las mujeres feministas son blancas, pero también hay mujeres negras, indias, $y$ un poco tocar el tema de la racialidad. (F6PH2)

De otro lado, también hay creaciones narrativas que centran la atención sobre "los rasgos occidentales" que predominan en el vídeo, reproduciendo al mismo tiempo ciertos estereotipos raciales y prejuicios etnocéntricos asociando una mayor presencia de la violencia machista con "lo musulmán" (en realidad una dimensión religiosa) y aseverando que en dichos contextos (se sobreentiende que se refiere al mundo árabe) las mujeres tienen mayores dificultades de acceso al trabajo y condición de vida:

Hemos estado hablando de que por ejemplo en la canción, las partes estaban reflejadas para personas más o menos de nuestros rasgos, pero por ejemplo los rasgos musulmanes y tipos como esos, se refleja más el machismo, porque suele haber en sitios como esos mucho más. Y las mujeres en lo social suelen optar a mucho menos cargos mayores. (F5PH2)

\title{
4. Discusión y conclusiones
}

Atendiendo a los resultados del análisis de los datos podemos establecer diversas consideraciones respecto de la potencialidad del Teatro Aplicado mediante la creación y presentaciones de una performance teatral para la prevención de la violencia de género y la promoción de la igualdad.

En primer lugar, el análisis actancial de esta pieza teatral, sus ejes narrativos y los roles asociados a las y los personajes pretenden señalar la vigencia y actualidad de la violencia de género entre jóvenes y adolescentes, sobre todo en determinados contextos vinculados al ocio y la socialización juvenil que se convierten en observatorios privilegiados como describe Sedano-Solis (2019) para diagnosticar y analizar los mecanismos coercitivos y encubiertos con los que se ejerce la dominación y la violencia de los hombres hacia las mujeres, prácticas que además son justificadas y toleradas socialmente en el marco de una cultura patriarcal hegemónica que funciona como mecanismo coadyuvante para anular la autonomía de las mujeres y su emancipación.

Pero al mismo tiempo, y a la luz de los resultados extraídos, hemos podido valorar en línea a lo que sostiene Pavis (2017) la potencialidad de los modelos de investigación basados en esquemas actanciales, también para rescatar toda una serie de micromachismos que la performance teatral no deja ver, quedan invisibilizados o excluidos de la propuesta narrativa. Parecen obviarse, pues, toda una serie de actividades vinculadas a los cuidados y servicios desarrolladas en el ámbito privado-doméstico mayoritariamente por las mujeres. Tampoco son contemplados los abusos sexuales explícitos del hombre en espacios públicos o privados, o la falta de diversidad racial en las mujeres representadas en las escenas teatrales y que sufren de episodios de violencia de género no sólo por "ser mujer" sino precisamente por ser "mujer racializada" examinados, por ejemplo, en Stahl (2018).

Es por eso que tanto las problemáticas (re)presentadas como aquellas otras que han sido invisibilizadas o no explicitadas en la performance, anticiparían en la audiencia un cambio en su rol y competencia es- 
pectatorial al crear una experiencia colectiva a nivel sensorial, afectivo y cognitivo en el que el alumnado de secundaria, en tanto hombres y mujeres jóvenes se ven representados e interpelados en sus diferentes posiciones y acciones respecto de la violencia micromachista, aspectos que han sido tratados también por Curnow (2013) o Beth (2018).

En segundo lugar, los resultados que hemos obtenido en la fase de dinamización de la performance teatral constatan los análisis desarrollados por Santos (2017) y Villanueva Vargas (2019) respecto del papel que juegan las metodologías dialógico-participativas para favorecer la inmersión e implicación del espectador en la problemática de la violencia de género y la promoción de la igualdad entre jóvenes y adolescentes. Efectivamente, estos procesos de mediación pedagógica, facilitación y reflexión de la obra se convierten en procesos culturalmente relevantes y pertinentes socialmente, al reflexionar de forma conjunta respecto de experiencias reales y auténticas vividas por el alumnado en relación con la violencia de género, que ha sido objeto de investigación en trabajos como los de Duffy y Powers (2018).

Por otra parte, al valorar y gestionar en el trabajo de mediación colectiva las distintas experiencias del alumnado de secundaria en relación con situaciones de micromachismo en la vida cotidiana, al problematizar con ellas y ellos en torno a diferentes cuestiones surgidas en la obra teatral respecto de la violencia patriarcal en sus contextos de socialización, vemos cómo se refuerza esa competencia espectatorial procurando, en línea a lo que argumenta Helbo (2021), no sólo un distanciamiento cognitivo respecto de la representación dramática sino, y sobre todo, confrontar y analizar (como también sostienen Cahill y Dadvand, 2020) los conocimientos tácitos que sobre el género trae el alumnado, convirtiéndoles en ese momento en audiencia implicada, confirmando así las propuestas performativas desarrolladas por Motos y otros (2013).

Son, por tanto, estos mecanismos de mediación una herramienta indispensable para desmontar, desestabilizar, deconstruir todas esas construcciones sociales opresivas respecto de las mujeres descritas en trabajos como los de Abraham (2017) o Bateman y Coetzee (2018), pudiendo favorecer entonces un cambio en las percepciones y/o acciones de la audiencia desde una perspectiva de género y promoción de la igualdad.

En tercer lugar, de los resultados destacados en la fase de (re)creación podemos evidenciar cómo ese proceso de construcción del espectador/a implica finalmente considerar a la audiencia de secundaria como actantes observadores/as, que no sólo justificarían y legitimarían la obra teatral a través de su presencia, mirada o escucha (fase de escenificación teatral), ni tampoco únicamente desde el involucramiento perceptivo y afectivo o el distanciamiento cognitivo (fase de dinamización). Lo que observamos también es que el proceso de performance teatral implica a su vez la necesidad de un proceso de enunciación colectiva, donde la sala y el escenario se inventan juntos, comparten actorialidad (Helbo, 2021), convirtiéndose el alumnado de secundaria en co-actores de un proceso, el de la performance, que deviene en un laboratorio de invención creativa, de búsqueda de otros sentidos posibles respecto de la violencia de género y los micromachismos por parte de jóvenes y adolescentes.

Las propuestas de modificación de la obra se materializan, como hemos visto, en torno a temáticas como el derecho a la emancipación de las mujeres (en sus acciones o en sus cuerpos), y la necesidad de establecer comportamientos igualitarios por parte de los varones para afrontar una nueva condición masculina. Este empoderamiento del alumnado busca la concreción de espacios libres de estereotipos de género, de las "obligaciones" patriarcales que dictan a los hombres y la creación de nuevas realidades no jerárquicas e igualitarias.

En el primer caso, las nuevas narrativas producidas por las mujeres adolescentes son traídas "a escena", teatralizadas y representadas a través de la voz, el gesto facial o la exposición pública de sus cuerpos, todo ello en un ambiente social protegido (procurado por la performance teatral) que permite, como ya ha señalado Coetzee (2018), que dicho conocimiento corporalizado se convierta, a través de la propia dinámica creativa, en memoria colectiva donde poder compartir las experiencias personales y ajenas vividas por mujeres adolescentes, sus miedos y sufrimientos, pero también sus expectativas de autonomía y liberación personal.

En el segundo caso, esas producciones narrativas implican que los adolescentes, en tanto hombres, puedan también denunciar que son víctimas del patriarcado, mostrando interés en un cambio hacia esas formas desiguales y violentas de relacionarse, convirtiéndose la performance teatral en una oportunidad para legitimar otros modelos alejados de los patrones de masculinidad tradicional. En la misma línea que sostienen Landy y Montgomery (2012), la experiencia ofrecida por la performance teatral sienta las bases para un cambio social, pues de forma simultánea, se convierte en una herramienta para la concientización de gé- 
nero, la promoción de la igualdad e incluso la revisión de las prácticas con las que varones adolescentes se relacionan con el colectivo de mujeres.

\section{Agradecimientos}

Esta investigación ha sido realizada en el marco del proyecto de investigación RTI2018-097144-B-IO0 NOMADIS: Nómadas del conocimiento: análisis de prácticas pedagógicas disruptivas en Educación Secundaria del Plan Nacional de I+D+i y del proyecto VA005G19 de la Consejería de Educación de la Junta de Castilla y León.

Esta investigación ha sido financiada por la Consejería de Educación de la Junta de Castilla y León a través de las ayudas destinadas a la contratación predoctoral de personal investigador, cofinanciadas por el Fondo Social Europeo (Orden EDU/556/2019)

\section{Referencias}

Abraham, N. (2017). Witnessing change: Understanding change in participatory theatre practice with vulnerable youth in a kids company-supported primary school. Research in Drama Education: The Journal of Applied Theatre and Performance, 22(2), 233-250. https://doi.org/10.1080/13569783.2017.1293510

Ballesteros, J. C., Sanmartín, A. y Tudela, P. (2018). Barómetro juvenil sobre vida y salud. Centro Reina Sofía sobre Adolescencia y Juventud. https://doi.org/10.5281/zenodo.3537684

Balme, C. (2013). Introducción a los estudios teatrales. Editorial Frontera Sur.

Bateman, T. y Coetzee, M. H. (2018). Dramatizing gender: Exploring gender constructs through drama-based strategies. Youth Theatre Journal, 32(2), 124-137. https://doi.org/10.1080/08929092.2018.1529470

Besselink, T. (2013). Learning choreography. En J. Moravec (Ed.), Knowmad society (pp. 89-128). Education Futures.

Beth, J. (2018). Towards recognition and regard: Creating connectivity in theatre education through intentional wakefulness. Youth Theatre Journal, 32(1), 30-44. https://doi.org/10.1080/08929092.2018.1445051

Bonino, L. (1995). Desvelando los micromachismos en la vida conyugal. En J. Corsi (Ed.), Violencia masculina en la pareja. Una aproximación al diagnóstico y a los modelos de intervención (pp. 191-208). Paidós.

Bonino, L. (1996). La violencia invisible en la pareja. En Consorci de Museus de la Generalitat Valenciana (Coord.), I Jornadas de género en la sociedad actual (pp. 25-45). Generalitat Valenciana.

Cahill, H. y Dadvand, B. (2020). Triadic labour in teaching for the prevention of gender-based violence. Gender and Education, 33(2), 252-266. https://doi.org/10.1080/09540253.2020.1722070

Coetzee, M. H. (2018). Embodied knowledge(s), embodied pedagogies and performance. South African Theatre Journal, 31(1), 1-4. https://doi.org/10.1080/10137548.2018.1425527

Curnow, J. (2013). Fight the power: Situated learning and conscientisation in a gendered community of practice. Gender and Education, 25(7), 834-850. https://doi.org/10.1080/09540253.2013.845649

Davids, T. y Willemse, K. (2014). Embodied engagements: Feminist ethnography at the crossing of knowledge production and representation. An introduction. Women's Studies International Forum, 43, 1-4.

https://doi.org/10.1016/j.wsif.2014.02.001

De Marinis, M. (2005). En busca del actory del espectador. Comprender el teatro II. Editorial Galerna.

De Toro, F. (1995). Towards an actantial model for theatre. En M. J. Valdes (Ed.), Theatre semiotics. Text and staging in modern theatre (pp. 129-142). Vervuert Verlagsgesellschaft. https://doi.org/10.31819/9783964563798-010

Díaz-Aguado, M.J., Martínez Arias, R., Martín Babarro, J. y Falcón, L. (2020). La situación de la violencia contra las mujeres en la adolescencia en España. Delegación del Gobierno contra la Violencia de Género. Ministerio de Igualdad.

Donoso, T., Rubio, M. y Vilà, R. (2018). La adolescencia ante la violencia de género 2.0: Concepciones, conductas y experiencias. Educación XX1, 21(1), 109-133. https://doi.org/10.5944/educxx1.20180 
Duffy, P. y Powers, B. (2018). Blind to what's in front of them: Theatre of the oppressed and teacher reflexive practice, embodying culturally relevant pedagogy with pre-service teachers. Youth Theatre Journal, 32(1), 45-59. https://doi.org/10.1080/08929092.2018.1445677

Gallagher, K. y Rodricks, D. (2017). Hope despite hopelessness: Race, gender, and the pedagogies of drama/applied theatre as a relational ethic in neoliberal times. Youth Theatre Journal, 31(2), 114-128. https://doi.org/10.1080/08929092.2017.1370625

Gallagher, K. y Sahni, U. (2019). Performing care: Re-imagining gender, personhood, and educational justice. Gender and Education, 31(5), 631-642. https://doi.org/10.1080/09540253.2019.1609652

García, A., Hidalgo, M., López, M. C. y Román M. R. (2018). Los micromachismos en los adolescentes. Su asociación con las relaciones de pareja y el modelo de maternidad y paternidad. Cultura de los Cuidados, 22(51), 144-153. https://doi.org/10.14198/cuid.2018.51.16

García-Huidobro, V., Del Canto, L., Sedano, A. y Compañía La Balanza. (2021). Teatro aplicado en educación. Ediciones Universidad Católica de Chile.

Greimas, A. (1971). Semántica estructural. Editorial Gredos.

Giambrone, A. (2016). Dramatic encounters: Drama pedagogy and conflict in social justice teaching [Tesis doctoral, Universidad de Toronto]. Archivo de la Universidad de Toronto. https://bit.ly/3aZdtmM

Helbo, A. (2013). Les Métamorphoses du spectateur. Dégrés, 161, 12-15.

Helbo, A. (2021). La metamorfosis del espectador y la competencia espectacular. Tropelías. Revista de Teoría de la Literatura y Literatura Comparada, 35, 11-22.

Landy, R. J. y Montgomery, D. T. (2012). Theatre for change: Education, social action and therapy. Palgrave Macmillan.

Mirza R. (2011). Teatro y representación: Perspectivas contemporáneas sobre teoría, historia y crítica del teatro latinoamericano y europeo. Universidad de La República.

Motos, T. Alfonso-Benlliure, V. y Fields, D. L. (2018). The impact of theatrical experiences on young adults in Spain. Research in Drama Education: The Journal of Applied Theatre and Performance, 24(2), 192-200. https://doi.org/10.1080/13569783.2018.1494562

Motos, T. y Ferrandis, D. (2015). Teatro aplicado. Octaedro.

Motos, T., Navarro, A., Ferrandis, D. y Stronk, D. (2013). Otros escenarios para el teatro. Ñaque.

Nicholson, H. (2005). Applied drama: The gift of theatre. Palgrave Macmillan. https://doi.org/10.1007/978-0-230-20469-0

Pavis, P. (2017). Diccionario del teatro: dramaturgia, estética, semiología. Paidós.

Román, R. y Faepb, A. (2017). Juventud universitaria, micromachismos y relaciones de noviazgo. REencuentro. Análisis de Problemas Universitarios, 28 (74), 149-169.

Sales Oliveira, C., Monteiro, A. A. y Ferreira, S. P. (2019). Gender consciousness through applied theatre. European Journal for Research on Education and Learning of Adults, 10(1), 77-92. https://doi.org/10.3384/rela.2000-7426.ojs352

Sánchez, M. C. (2008). Informática y análisis cualitativo. En M. A. Verdugo, M. Crespo, M. Badía y B. Arias (Coords.), Metodología en la investigación sobre discapacidad. Introducción al uso de las ecuaciones estructurales (pp. 127-177). INICO.

Santos, B. (2017). Teatro del oprimido. Raíces y alas: Una teoría de la praxis. Descontrol.

Sedano-Solís, A. S. (2019). El teatro aplicado como campo interdisciplinario de investigación en los Estudios Teatrales. Artnodes, 23, 104-113. https://doi.org/10.7238/a.v0i23.3260

Stahl, S. (2018). Acting out to call in. Practicing theatre of the oppressed with high school students. The Educational Forum, 82(3), 369-373. https://doi.org/10.1080/00131725.2018.1457123

Stake, R. E. (1999). Investigación con estudio de casos. Morata. 
Taylor, L. (2019). Towards a concept of inefficiency in performance and dialogue practice. Research in Drama Education: The Journal of Applied Theatre and Performance, 24(3), 333-351.

https://doi.org/10.1080/13569783.2019.1619449

Ubersfeld, A. H. G. (1999). The actantial model in theatre. En A. H. G. Ubersfeld (Ed.), Reading theatre (pp. 32-71). University of Toronto Press. https://doi.org/10.3138/9781442679023-005

Villanueva Vargas, M. C. (2019). Opening spaces for Critical Pedagogy through Drama in Education in the Chilean classroom. [Tesis doctoral, Trinity College Dublin]. Archivo del Trinity College Dublin.

\section{Breve CV de los/as autores/as}

\section{Rocío Anguita-Martínez}

Profesora Titular de Universidad del Dpto. de Pedagogía. Facultad de Educación y Trabajo Social de la Universidad de Valladolid. Directora del Grupo de Investigación Reconocido (GIR) CEAEX (Ciudadanía, Ecologías del Aprendizaje y Educación Expandida). Mi labor investigadora se ha desarrollado a lo largo de este tiempo, con la participación en el contexto de 29 proyectos obtenidos de forma ininterrumpida desde 1990 hasta la actualidad en convocatorias competitivas. He publicado más de 30 artículos en revistas indexadas, además de 5 libros, 15 capítulos de libro y 20 contribuciones en proceedings nacionales e internacionales. relacionadas con los campos de la educación y las TICs y la igualdad de las mujeres en los sistemas educativos. Email: rocio.anguita@uva.es

ORCID ID: https://orcid.org/0000-0002-2533-8871

\section{Yasna Pradena-García}

Investigadora predoctoral, por la Consejería de Educación de la Junta de Castilla y León y el Fondo Social Europeo, en la Universidad de Valladolid (UVA), Facultad de Educación y Trabajo Social, Departamento de Pedagogía. Estudiante, en la misma universidad, del Doctorado de Investigación Transdisciplinar de Educación desarrollando la tesis "Teatro Aplicado para la prevención de la violencia de género y la promoción de la igualdad: Un estudio de caso en el ámbito de la Formación Profesional (FP)". He participado como investigadora en el estudio de caso "Artivismo para aprender igualdad en dos ciclos superiores de FP" del proyecto "NOMADIS: Nómadas del conocimiento: análisis de prácticas pedagógicas disruptivas en Educación Secundaria” con el Grupo de Investigación Reconocido (GIR) CEAEX (Ciudadanía, Ecologías del Aprendizaje y Educación Expandida). Email: yasnapatricia.pradena@uva.es

ORCID ID: https://orcid.org/0000-0001-7687-6592

\section{Eduardo Fernández-Rodríguez}

Profesor Contratado Doctor del Dpto. de Pedagogía. Facultad de Educación de Palencia. Universidad de Valladolid. Miembro del Grupo de Investigación Reconocido (GIR) CEAEX (Ciudadanía, Ecologías del Aprendizaje y Educación Expandida). Fruto de mi actividad investigadora, he desarrollado trabajos de distinta naturaleza en revistas nacionales e internacionales indexadas con la participación en el contexto de 8 proyectos obtenidos en convocatorias competitivas: 8 nacionales y 2 regionales, con la publicación de 30 artículos en revistas indexadas relacionadas con los campos del currículum, el análisis político de los discursos educativos, formación del profesorado, educación y alfabetización mediática, educación y tics, pedagogías críticas, además de 4 capítulos de libro y 10 contribuciones en Proceedings y libros de actas nacionales e internacionales.Email: eduardo.fernandez@uva.es

ORCID ID: https://orcid.org/0000-0001-8611-2510 